Author Accepted Version. Final version published as: Temple, J. B., Kelaher, M., \& Williams, R. (2019). Disability discrimination and avoidance in later life: prevalence, disability differentials and association with mental health. International Psychogeriatrics, 31(9), 13191329.

Disability Discrimination and Avoidance in Later Life: Prevalence, Disability Differentials and Association with Mental Health

\author{
Jeromey Temple \\ Associate Professor \\ Demography and Ageing Unit \\ Melbourne School of Population and Global Health, \\ University of Melbourne \\ Australia \\ Margaret Kelaher \\ Professor \\ Centre for Health Policy \\ Melbourne School of Population and Global Health, \\ University of Melbourne \\ Australia \\ Ruth Williams \\ Academic Convenor \\ Demography and Ageing Unit \\ Melbourne School of Population and Global Health, \\ University of Melbourne \\ Australia
}

Please direct correspondence to Jeromey B. Temple, Associate Professor, Melbourne School of Population and Global Health, 207 Bouverie St, University of Melbourne, Melbourne, VIC, 3010 Australia (Email: Jeromey.Temple@unimelb.edu.au Phone: +61 3 90359900). 


\section{Disability Discrimination and Avoidance in Later Life: \\ Prevalence, Disability Differentials and Association with Mental Health}

\section{Abstract}

Background: Later life is a period of increased risk of disability, but there is little quantitative evidence regarding the exclusion of older people (through discrimination and avoidance) due to their health conditions. This study aims to (1.) measure the prevalence of disability exclusion in later life, (2.) examine how experiences of exclusion differ by disability type, and (3.) investigate the association of exposure to exclusion with psychological distress.

Methods: Using data from the 2015 ABS Survey of Disability, Ageing and Carers, we calculated the prevalence of people aged 55 years and over with a disability experiencing discrimination and engaging in avoidance behaviours, disaggregated by 18 detailed disability types. Modified Log-Poisson models were fitted to estimate Prevalence Ratios to measure the association between exclusion and psychological distress, stratified by disability type.

Results: In 2015, about $5 \%$ of Australians aged 55 years and over with a disability reported experiencing an instance of disability discrimination, and one in four reported avoiding a situation or context due to their disability. Accounting for psychosocial comorbidities and with extensive demographic controls, exposure to disability avoidance $(\mathrm{PR}=1.9,95 \% \mathrm{Cl} 1.7,2.1)$ or discrimination $(\mathrm{PR}=1.7,95 \% \mathrm{Cl} 1.4,2.1)$ almost doubled the probability of experiencing psychological distress. Effects were heightened for individuals reporting specific disabilities including sensory and speech and physical disabilities as well as those reporting a head injury, stroke or acquired brain injury.

Conclusions: Despite protections against disability discrimination in legislation, discrimination and avoidance due to disability is prevalent and is associated with poor mental health outcomes.

Keywords: Disabilities, Discrimination, Avoidance, Psychological Distress. 


\section{Disability Discrimination and Avoidance in Later Life: \\ Prevalence, Disability Differentials and Association with Mental Health}

\section{Introduction}

Later life is a period of increased risk of disability and comorbidity (WHO, 2011; Kraus, 2017). Between $35 \%$ and $50 \%$ of the population aged 65 years and over are estimated to be living with a disability in the UK, US and Australia (ABS, 2016; Department for Work and Pensions, 2016; Kraus, 2017). Given the inexorable population ageing that is occurring across most high-income countries, the number and proportion of people living with a disability is likely to exhibit considerable growth over coming decades (Guzman-Castillo et al., 2017; WHO 2012). For example, in the UK, it is estimated that the number of older people with a disability will increase by around 40\% between 2002 and 2022 (Papworth Trust, 2016). In Australia, the number of older Australians living with a profound disability is projected to increase by $70 \%$ over the period 2006 to 2031 (Giles et al., 2003).

In this context, Governments have sought to plan for the burgeoning health needs of this growing demographic. For example, in Australia the NDIS (National Disability Insurance Scheme - launched in stages from 2016), seeks to improve access to mainstream and community services with a view to improving the health and wellbeing of people living with a disability. Many OECD countries are also increasingly altering their disability policies from passive support to an orientation of active employment and inclusion (OECD, 2008). While this is laudable, one aspect of the exclusion of this group that has garnered less attention is how experiences of discrimination or avoidance of health care and other settings due to disability, is associated with health and wellbeing. This is despite considerable agreement in the literature that exposure to discrimination in its many guises is an important social determinant of health, with 
studies underscoring the implications for deleterious mental health outcomes (Kessler et al., 1999; Paradies et al., 2015; Lyons et al., 2017).

Indeed, recent studies have noted the need for more research on ageism and discrimination in later life, pointing to the considerable health consequences and interrelationship with human rights (Sargent-Cox, 2017; Biggs et al., 2013). Recent studies have also documented age based discrimination in health care contexts such as long-term care (San Jose et al., 2017) and the role of health care staff such as nurses and physicians (Schroyen et al., 2016; Werner et al., 2008). Discrimination in these settings is oftentimes more pronounced for marginalised groups such as LGTBI people (Tinney et al., 2015). Age discrimination too has been shown to be particularly prevalent in the workplace context in Australia. A national prevalence study of age discrimination in the workplace by the Australian Human Rights Commission found that $27 \%$ of Australians aged 50 and over had experienced discrimination recently, with one third of these experiences occurring at the application and recruitment stage (AHRC, 2015).

Despite this growing literature, there is a paucity of evidence on the relationship between experiencing discrimination due to a disability and psychological distress, specifically during later life. A few studies have examined disability discrimination and psychological distress within the broader population. Wamala et al.'s (2007) Swedish study found for people aged 18-84 years, exposure to disability discrimination increased the odds of psychological distress by 1.65 times (OR 95\% Cl 1.26 to 2.17 ). Temple and Kelaher's (2018) nationally representative study of people aged 15 years and over with a disability in Australia, mirrored these findings. They found experiencing disability discrimination increased the odds of psychological distress by 2.2 times (OR $95 \% \mathrm{Cl} 1.74$ to 2.26 ). Another Australian study of the working age population (aged 15-64) found disability discrimination increased the odds of psychological distress as 
well as poorer self-rated health (Krnjacki et al., 2018). More generally, disability discrimination has been found to be associated with new or worsening disabilities and avoidance of health care in US, South American and Australian studies (Rogers et al., 2015; Mosco-Porras, 2017; Temple et al., 2018). To date there have been no studies specifically examining differences in exposure to discrimination and its association with psychological distress stratified by different disability types. This is despite literature citing that people with a communication, physical or intellectual disability are at greater risk of avoiding or not seeking care specifically due to perceived discrimination (Moscoso-Porras, 2018; Backer et al., 2009; Ali et al., 2013).

In this study, we seek to complement the extant literature by examining how disability discrimination and avoidance of situations or contexts due to disability are associated with psychological distress during later life. Specifically, we seek to answer three questions. Firstly, what is the prevalence of self-reported disability discrimination and disability related avoidance by older Australians? Secondly, do experiences of discrimination and avoidance differ by an individual's disability type? Finally, is exclusion (either discrimination or avoidance) differentially associated with psychological distress in later life by disability type?

\section{Methods}

\section{Data}

Data for this study are from the 2015 Survey of Disability, Ageing and Carers (SDAC) conducted between July and December 2015. Using multi-stage sampling techniques, individuals living in private dwellings, in self-care retirement villages and in care accommodation were included in the survey. The public use data file included survey weights to account for differences in the respondents' chance of selection into the survey. The weights were calibrated against estimated population counts by the ABS to correct for over or under enumeration of particular demographic groups, which may 
occur due to random sampling or non-response (ABS, 2016). This study utilises information collected from the household component of the survey. Of the total 31,957 private dwellings contacted, 25,555 fully responded, yielding a response rate of $80 \%$.

The module on discrimination and avoidance was included in a personal interview for all people aged 15 years and over with a disability who lived in households. The final sample consisted of 6,492 people aged 55 years and over with a disability and living in private residences. The cut off age of 55 is chosen for two reasons. Firstly, Australian studies oftentimes define the older population as those aged 55 and over as from this age, citizens are legally entitled to access private superannuation and retire (for those born prior to 1960). Secondly, the age group 55-59 is an important turning point in the prevalence of disability in Australia. In 2015, approximately $18 \%$ of the population were estimated to be living with a disability (ABS, 2016). From age 55-59, this is the first age group in the population with a prevalence rate of disability significantly higher than the population average $(23.4 \%)$. The proportion of the population with a disability then rises to $37.8 \%$ at ages $65-69$, then steeply to $52.9 \%$ at ages $75-79$ and to $85.4 \%$ for persons aged 90 and over (ABS, 2016).

\section{Measures of Discrimination and Exclusion}

Cross sections of SDAC have previously been conducted by the ABS periodically from 1981. In the latest 2015 cross section, the ABS included detailed measures on discrimination and avoidance due to a person's disability. Respondents were asked "In the last 12 months do you feel that you have experienced discrimination or have been treated unfairly by others because of your condition/s?" For avoidance, respondents were asked "In the last 12 months have you avoided situations because of your condition(s)?" 
Measurement of Psychological Distress and Psychosocial Disability

The SDAC also included measures of psychological distress. The specific measure of psychological distress used was Kessler's K10. The K10, although not a diagnostic tool, is a widely used screening instrument for psychological distress based on the respondent's emotional state in the four weeks prior to the interview (ABS, 2016; Anderson et al., 2013). Following examples in the literature as well as recommended clinical guidelines, we indicate a 'high' or 'very high' score on Kessler's measure as indicating psychological distress (Kelaher et al., 2014; ABS, 2014). In the Australian case, the $\mathrm{K} 10$ as a screening tool is preferred over other measures such as the $\mathrm{GHQ}$ or SF-12 (Andrews et al., 2001).

\section{Measures of Disabilities}

In addition to these new measures on discrimination and psychological distress, the SDAC instrument collected detailed measures of the recipient's disabling conditions. These consisted of 16 individual disabling conditions (see Table 1), which broadly defined constituted six aggregate categories including sensory and speech, intellectual, physical, psychosocial, head injury and other disabilities. The ABS definition of disability is "any limitation, restriction or impairment which restricts everyday activities and has lasted, or is likely to last, for at least six months" (ABS, 2016).

For the first time in 2015, the SDAC also measured psychosocial disabilities. This concept is broader than a psychological disability and encompasses nervous or emotional conditions, memory problems or confusion, social or behavioral difficulties, a mental illness for which help or supervision is required and brain injuries including stroke, that lead to mental illness or other cognitive problems (ABS, 2016). This concept was designed by the ABS to specifically measure conditions that "have an 
impact on a person's ability to participate fully in daily living and opportunities like education, employment, and social and cultural activities" (ABS, 2016).

\section{Statistical Model}

To examine the association between exposure to disability exclusion and psychological distress, several estimation issues needed to be addressed. Firstly, as the dependent variable was psychological distress, there was a possibility of endogeneity or confounding effects between reports of distress by those with psychosocial disabilities. Our approach to this issue was to split the sample into (1.) the full population, and (2.) the population excluding psychosocial disabilities. We presented results for both populations for transparency. To examine variations in the association between disability exclusion and psychological distress by disability type, we further split the sample by each of the defined 16 disability types and six aggregate disability categories.

Secondly, the prevalence of psychological distress in this sample was relatively high for specific disabling conditions. The empirical literature on disability discrimination and methodological literature on modelling binary outcomes raises concerns about overestimation of odds ratios in these circumstances, (Barros and Hirakata, 2003; Moscoso-Porras, 2018). Again, following examples in the literature we fit Zou's modified log-Poisson models to estimate Prevalence Ratios to measure the association between disability exclusion and distress (Moscoso-Porras, 2018; Zou, 2004). Specifically, this technique utilises a Poisson variance and log link function with a robust variance-covariance matrix of estimators, optimised by maximum likelihood (Zou, 2004).

Using the raw modified log-Poisson coefficients, we calculated two parameters of interest. Firstly, we calculated prevalence ratios (PR) which measured the change in 
the probability of psychological distress given a change in exposure to discrimination or avoidance, once all other factors in the model were controlled for. Control variables included age, sex, country of birth (Australia, English speaking countries, Non-Englishspeaking country), social marital status (married, separated, divorced, widowed, never married), region of residence (major cities, inner regional areas, other) and education (degree or above, certificate, school only). For the two models of avoidance and discrimination in the population with all disabilities, we also included controls for disability type. Secondly, to complement and provide a balanced interpretation of the PR coefficients, we also presented the marginal effects (ME) of the probability of distress, relative to exposure to discrimination or avoidance. Specifically, the ME estimates showed the difference in the probability of distress between the group reporting having experienced discrimination and those who do not report experiencing discrimination, along with the statistical significance of the difference. This is necessary as we observed considerable differences in the prevalence of distress by disability type. The marginal effects were calculated with controls for all other factors in the model.

\section{Results}

Table 1 displays the percentage of respondents exposed to discrimination, avoidance and psychological distress, disaggregated by disability type. The majority of older Australians with a disability reported a physical disability (73\%) in 2015 . Just under half reported a sensory or speech disability $(40 \%)$, or other disability type $(42 \%)$. About $12 \%$ had a psychosocial disability in 2015 .

In the full sample, about $4.5 \%$ of older people with a disability reported an instance of discrimination specifically due to their health condition. Importantly, we observed variations in the proportions reporting these measures of exclusion by disability type. Levels or reported discrimination were heightened for those with psychosocial 
conditions (13.6\%), head injuries (11.27\%) or intellectual disabilities (12.3\%). Although about $5 \%$ of individuals with any physical disability reported discrimination, levels were elevated for people who experience blackouts or seizures $(10.5 \%)$ and people with incomplete use of their arms or fingers (8.8\%) or feet or legs (8.3\%).

In contrast, levels of avoidance were significantly higher, with $25 \%$ of respondents with a disability reporting avoidance. Again, there were strong variations in reports by disability type, with individuals with a psychological condition significantly more likely to report avoidance $(57.6 \%)$, followed by people with an intellectual disability $(47.4 \%)$ or head injury (44.6\%). In excess of one third of respondents with specific physical or other disabilities also reported instances of avoidance. Among respondents with sensory and speech disabilities, those with speech difficulties are more likely to report discrimination $(9 \%)$ or avoidance $(40.1 \%)$.

Just under 1 in 4 people with a disability were in psychological distress in 2015 (23.6\%). As expected, respondents with psychological disabilities were far more likely to be in distress (64\%) as were those with head injuries $(41 \%)$ and intellectual disabilities (49\%). For these later two groups, there was a high prevalence of a psychosocial comorbidity (51\% and $47 \%$ for intellectual disability and head injuries respectively). People with speech difficulties (42\%) and breathing difficulties (44\%) were again highlighted as groups at a heightened risk of poor mental health outcomes.

To control for the effect of psychosocial comorbidities on reports of distress, we also estimated the prevalence of distress within a restricted sample of respondents without a psychosocial disability. As expected, the prevalence of distress fell across all major categories; for sensory and speech $(18.8 \%$ to $14.4 \%)$, intellectual disability ( $49 \%$ to $28.9 \%)$, physical disability ( $27.8 \%$ to $21.5 \%)$, head injury $(41.4 \%$ to $28.2 \%)$ and other disability types (23.6\% to $18 \%)$. Notwithstanding, these rates remained considerably 
above the population level prevalence of psychological distress (9.2\%) among nonIndigenous Australians aged 55 years and over (ABS, 2014).

\section{[TABLE 1]}

Although these differences point to a potential association between discrimination and psychological distress, it was important to control for factors that were associated with distress. Moreover, as evident by the strong presence of psychosocial comorbidities for a number of disability types, it was important to split the sample into those with and those without psychosocial disabilities. Table 2 presents results from the modified logPoisson models of discrimination and distress, stratified by impairment type. In both the full (PR 1.5 95\% Cl 1.3 1.6) and restricted samples (PR $1.795 \% \mathrm{Cl} 1.42 .1$ ), discrimination was associated with about a 1.5 to 1.7 fold increase in the probability of distress. For those exposed to discrimination, the probability of distress as measured by the marginal effect was $0.1(p<0.001)$ to $0.12(p<0.001)$ higher than individuals with no exposure to discrimination. This change in probability was from an average probability of distress of about 0.23 in the full sample and was thus a considerable change.

In the full sample, across all aggregate disability categories, discrimination was associated with distress. At the aggregate disability level, the association between discrimination and distress ranged from sensory and speech disability (PR 2.99 95\% Cl $2.45,3.66)$ with a marginal effect of $0.34(p<0.001)$ to any psychosocial disability $(P R$ $2.9995 \% \mathrm{Cl} 2.45,3.66)$ with a marginal effect of $0.2(p<0.001)$. When interpreting these results, it is important to note the higher prevalence of psychological distress among those with a psychosocial disability. When restricting the sample to those without a psychosocial comorbidity, the results were broadly replicated. However, for three specific conditions (difficulty learning or understanding, blackouts, seizures or loss of consciousness, and disfigurement and deformity), the parameter coefficients lost 
strength and were not statistically significant at the $95 \%$ level. This was not unexpected as all three groups (1.) have a small sample size in the full sample, (2.) have high levels of psychosocial comorbidities, and (3.) thereby lose a significant portion of their sample size. In the restricted sample, each group had between 100-200 respondents. For example, for difficulty learning or understanding, approximately $(n=263$ in the full sample), $51 \%$ had a psychosocial comorbidity. This reduced the sample size to just $\mathrm{n}=124$ in the restricted sample.

\section{[TABLE 2]}

Consistent with the results for discrimination, avoidance was also strongly associated with distress (Table 2$)$ in both the full sample ( $P R=1.72,95 \% \mathrm{Cl} 1.58,1.88)$ and restricted sample of people without a psychosocial disability $(P R=1.89,95 \% \mathrm{Cl} 1.69$, 2.12). In both samples, avoidance was associated with distress for each disability type. The association of avoidance with distress was particularly strong (with PRs above 2) for sensory and speech $(\mathrm{PR}=2.9795 \% \mathrm{Cl} 2.55,3.45)$ and physical disabilities $(P R=2.3595 \% \mathrm{Cl} 2.15,2.56)$. The marginal effects ranged from $0.29(p<0.001)$ for intellectual disabilities to $0.187(p<0.001)$ for psychosocial disabilities in the full sample. Both groups had a high underlying prevalence of distress.

\section{[TABLE 3]}

Summarising results from tables 2 and 3, there were a number of aggregate and detailed disability types for which the probability of distress shifted by approximately 0.2 given an exposure to both discrimination and avoidance. These included sensory and speech, physical disability, and head injury, stroke or acquired brain injury. The last two of these categories exhibited higher levels of underlying prevalence of psychological distress. Using the finer measures of disabilities, people with loss of hearing, breathing difficulties, difficulty gripping or holding things or restrictions in physical activities roughly fell into this category also. Within the full sample (including 
respondents with a psychosocial disability), with the exception of people who reported a nervous or emotional condition, the change in probability of distress given exposure to discrimination or avoidance was approximately 0.2 or above for all categories including mental illness, memory problems or periods of confusion, or those with social or behavioural difficulties.

\section{Discussion}

Australia has a strong legislative framework to protect people with a disability from discrimination. At the international level, Australia ratified the United Nations Convention on the Rights of Persons with Disabilities in 2008. At the Federal level, the Disability Discrimination Act 1992 seeks to ensure equal rights, opportunities and access for people with a disability. Furthermore, various state and territory level antidiscrimination laws further prohibit discrimination based on a person's disability or health condition. Notwithstanding these legal protections and agreements with international accords, results presented in this study show that a sizeable minority of older Australians with a disability report having experienced either discrimination (5\%) or avoidance $(25 \%)$ of activities and situations due to their health conditions.

We also demonstrated that reports of discrimination were heightened among older Australians with psychosocial, intellectual disabilities and head injuries. Almost half of individuals with an intellectual disability or head injury concurrently had a psychosocial disability. Our results mirror that of the wider literature which shows that people with intellectual disabilities are more likely to be diagnosed with depression compared with the general community (Department of Health 2011), and more likely to report discrimination. An Australian study of community attitudes found that the majority of respondents thought people with intellectual, learning, psychological or psychiatric disabilities were unable to fully contribute to society (ACT Disability Advisory Council 2004). In addition, adults with a mental illness are nearly four times more likely to 
experience violence (Hughes et al. 2012); while other statistics indicate that 90 per cent of women with intellectual disabilities have been sexually abused (Frohmader 2002). Our findings, combined with those in the literature, demonstrate the stark reality of the vulnerability of this group in experiencing discrimination, violence and abuse.

Our results also show avoidance and discrimination were also elevated for people with specific physical disabilities such as blackouts/seizures, incomplete use of arms and fingers or legs and feet. These findings mirror those from a Peruvian study which found that the association of discrimination with healthcare seeking behaviour was particularly pronounced for those with physical disabilities (Moscoso-Porras and Alvarado, 2018). The Australian Human Rights commission notes complaints regarding physical disability and discrimination are dominated by issues with employment and the workplace (AHRC, 2016b). These largely comprise of physical mobility disability in relation to access barriers in the workplace and the unwillingness of employers to make reasonable workplace adjustments. Unemployment and the association with poor mental health is well documented, as is the correlation between experiences of discrimination and poor mental health (Paul et al., 2009; Pascoe et al., 2009). The result of experiencing both unemployment and discrimination is likely to have a compounded effect on psychosocial distress on people with disability and the likely behaviours associated with avoidance.

These levels of reported discrimination and avoidance are important to the health and wellbeing of older Australians. As we illustrated, exposure to either discrimination or avoidance is associated with an increased probability of psychological distress. This relationship holds even when comorbidities with psychosocial disability are removed from the analysis. These empirical results underscoring the association of disability exclusion with psychological distress are consistent with a large body of literature in the field of discrimination and health more generally. Over time, these studies have 
developed a framework to understand the effects of avoidance and discrimination on health, particularly as it relates to racial discrimination (Paradies et al., 2015). Racism can result in negative health impacts through several key pathways including by: (1) increasing stress; (2) decreasing health promoting behaviours (e.g. physical activity); (3) decreasing health-damaging behaviours (e.g. alcohol and drug use); (4) reducing access to key health-promoting resources (e.g. employment, education, health and aged care services etc.); (5) increasing dysregulation (e.g. sleep disruption); and (6) increased health-damaging exposures (e.g. toxic substances) (Paradies et al., 2015). Avoidance is recognised as a pathway via which discrimination can have deleterious effects either through reducing access to health promoting resources or increasing risky behaviour (Kelaher et al., 2014; Han et al., 2015). However the links between avoidance and health have rarely been explored independently. Limiting access to health promoting facilities and resources is a key way in which discrimination against people with disabilities is enacted. Developing a better understanding of the nature of and health effects of avoidance is crucial to developing appropriate strategies to address these issues among people with disabilities.

We further showed that although discrimination and avoidance was associated with distress across each broad disability type, the effects were more pronounced for some specific disability types. These included groups with average or lower than average levels of psychological distress (sensory and speech), and groups with high prevalence rates of distress (specific physical disabilities as well as people with a head injury, acquired brain injury or other disability type). These findings demonstrate that avoidance and discrimination are important for all people with disabilities. High level of avoidance and discrimination among people with psychosocial and intellectual disabilities highlight the importance of going beyond addressing physical accessibility to considering changing societal norms. 


\section{Limitations and Extensions}

In interpreting our results, it is important to consider the limitations of the data and design. The SDAC are cross sectional data and we cannot attribute a causal relationship between discrimination or avoidance and psychological distress. The measures were also self-reported and estimates of discrimination may be biased as (1.) some respondents may feel uncomfortable disclosing instances of discrimination or avoidance, or (2.) some self-reported instances of exclusion may be perceived only. Moreover, the focus of this study has been on interpersonal discrimination, but experiences of structural discrimination are also likely to be associated with poor mental health outcomes.

These limitations suggest a number of pathways for future research. There is considerable need for longitudinal analysis of the pathways between onset of disability, exposure to discrimination and psychological distress. Unfortunately, there remains a lack of measures of disability discrimination and psychological distress more generally in nationally representative datasets in Australia. A further limitation is the relatively small sample size for specific disability types when examined for older Australians. This further raises the question of the generalisability of our findings to younger age groups where disabilities may be less common and discrimination potentially more pronounced.

\section{Conclusions}

Noting these limitations and extensions, results from this study showed that a sizeable minority of people with a disability reported experiencing instances of discrimination or avoidance due to their health condition. We further showed that such exposure was associated with an increased probability of psychological distress and that the impacts of this exposure were heightened for respondents with specific disabilities such as sensory and speech disability (with a lower overall probability of distress) and specific 
physical disabilities as well as those with a head injury, acquired brain injury or other disability type (with an overall higher level of psychological distress). With the continued rollout of the NDIS in Australia and increasing growth of the number of older Australians living with a disability due to population ageing, addressing and promoting the inclusion of people with a disability is a pressing national priority.

\section{Conflict of Interest}

None.

\section{Description of Author Roles}

J. B. Temple, M. Kelaher and R. Williams jointly designed the study and authored the manuscript. J. B. Temple performed the data analysis. All authors read the final version of the manuscript.

\section{Acknowledgement}

The Australian Bureau of Statistics (ABS) made data for this study available to the authors. The Australian Research Council's (ARC) Centre of Excellence in Population Ageing Research (CE1101029) funds author 1. The opinions expressed herein do not reflect those of the ABS or ARC.

\section{References}

ACT Disability Advisory Council. (2004). Snapshot of community attitudes on disability in the ACT. Canberra: ACT Disability Advisory Council.

Ali, A., Scior, K., Ratti, V., Strydom, A., King, M. and Hassiotis, A. (2013). Discrimination and other barriers to accessing health care: Perspectives of patients with mild and moderate intellectual disability and their carers. PLOS one, 8(8): e70855. https://doi.org/10.1371/journal.pone.0070855 
Anderson, T., Sunderland, M., Andrews, G., Titov, N., Dear, B. and Sachdev, P. (2013). The 10-Item Kessler Psychological Distress Scale (K10) as a Screening Instrument in Older Individuals. American Journal of Geriatric Psychiatry, 21(7), 596606.

Andrews, $\mathrm{G}$ and Slade, T. Interpreting scores on the Kessler Psychological Distress Scale (K10). Australian and New Zealand Journal of Public Health, 25, 494-7.

Australian Bureau of Statistics. (2016). Disability, ageing and carers, Australia: Summary of findings, Catalogue Number 4430.0. Canberra: Australian Bureau of Statistics.

Australian Bureau of Statistics. (2014). Australian Aboriginal and Torres Strait Islander Health Survey: First results, 2012-13 Australia, Catalogue Number 47270D007_20122013. Canberra: Australian Bureau of Statistics.

Australian Human Rights Commission. (2015). National prevalence survey of age discrimination in the workplace. Sydney: Australian Human Rights Commission.

Australian Human Rights Commission. (2016a). Access for all: Improving accessibility for consumers with disability. Sydney: Australian Human Rights Commission

Australian Human Rights Commission (2016b). Willing to Work: National inquiry into employment discrimination against older Australians and Australians with disability. Sydney: Australian Human Rights Commission 
Backer, C., Chapman, M. and Miller, D. (2009). Access to secondary healthcare for people with intellectual disabilities: A review of the literature. Journal of Applied Research in Intellectual Disabilities, 22, 514-525

Barros, A. and Hirakatam, V. (2003). Alternatives for logistic regression in crosssectional studies: an empirical comparison of models that directly estimate the prevalence ratio. BMC Medical Research Methodology, 3, 21.

Belsley, D., Kuh, E. and Welsch, R. (1980). Regression diagnostics: Identifying influential data and sources of collinearity. New York: John Wiley \& Sons, Inc.

Biggs, S., Haapala, I. (2013). Elder mistreatment, ageism and human rights. International Psychogeriatrics, 25(8), 1299-1306.

Department of Health. (2009). Victorian population health survey of people with an intellectual disability. Melbourne: Victorian Department of Health.

Department for Work and Pensions, Family Resources Survey 2015/16, https://assets.publishing.service.gov.uk/government/uploads/system/uploads/attachm ent data/file/600465/family-resources-survey-2015-16.pdf

Frohmader, C. (2002). There is no justice - THERE'S JUST US. The status of women with disabilities in Australia. Canberra: Women with Disabilities Australia.

Giles, L., Cameron, I and Crotty, M. (2003). Disability in older Australians: Projections for 2006-2031. Medical Journal of Australia, 179, 130-133. 
Guzman-Castillo, M., Ahmadi-Abhari, S., Bandosz, P., Capewell, S., Steptoe, A., Singh-Manoux, A., Kivimaki, M., Shipley, M., Brunner, E and O’Flaherty, M. (2017). Forecasted trends in disability and life expectancy in England and Wales up to 2025: a modelling study. Lancet Public Health, 2, e307-13 http://dx.doi.org/10.1016/ S24682667(17)300919

Han, C., Ayala, G., Paul, J., Boylan, R., Gregorich, W and Choi, K. (2015). Stress and coping with racism and their role in sexual risk for HIV among African American, Asian/Pacific Islander, and Latino men who have sex with men. Archives Of Sexual Behavior, 44(2), 411-420.

Hughes, K., Bellis, M., Jones, L., Wood, S., Bates, G., Eckley, L., McCoy, E., Mikton, C., Shakespeare, T. and Officer, A. (2012). Prevalence and risk of violence against adults with disabilities: a systematic review and meta-analysis of observational studies, Lancet, 379(9826), 1621-9.

Kelaher, M.A., A.S. Ferdinand, and Y. Paradies. (2014). Experiencing racism in health care: the mental health impacts for Victorian Aboriginal communities. Medical Journal of Australia, 201(1), p. 44-7.

Kessler, R., Mickelson, K. and Williams, D. (1999). The prevalence, distribution and mental health correlates of perceived discrimination in the United States. Journal of Health and Social Behavior, 40, 208-230.

Kraus, L. (2017). 2016 Disability Statistics Annual Report. Durham: University of New Hampshire. 
Krnjacki, L., Priest, N., Aitken, Z., Emerson, E., Llewellyn, G., King, T and Kavanagh, A. (2018). Disability-based discrimination and health: Findings from an Australianbased population study. Australian and New Zealand Journal of Public Health, 42(4):172-4.

Lyons, A., Alba, B., Heywood, W., Fileborn, B., Minichello, V., Barrett, C., Hinchliff, S., Malta, S. and Dow, B. (2017). Experiences of ageism and the mental health of older adults. Aging and Mental Health, 10, 1-9. doi: 10.1080/13607863.2017.1364347.

Moscoso-Porras. M. and Alvarado, G. (2018). Association between perceived discrimination and healthcare seeking behavior in people with a disability. Disability and Health Journal, 11(1), 93-98. doi: 10.1016/j.dhjo.2017.04.002

National People with Disabilities and Carer Council. (2009). Shut out: the experience of people with disabilities and their families in Australia. Canberra: Department of Families, Housing, Community Services and Indigenous Affairs.

OECD (2008). Modernising sickness and disability policy: OECD thematic review on sickness, disability and work issues paper and progress report. Available from http://www.oecd.org/els/emp/40495992.pdf, accessed 17/04/18.

Papworth Trust. (2016). Disability in the United Kingdom. Available at http://www.papworthtrust.org.uk/sites/default/files/Disability\%20Facts\%20and\%20Fig ures $\% 202016$.pdf, accessed 17/04/18.

Paradies, Y., Ben, J., Denson, N., Elias, A., Priest, N., Pieterse, A., Gupta, A., Kelaher, M. and Gee, G. (2015). Racism as a determinant of health: A systematic review and meta-analysis. Plos One, 10(9), e0138511-e. 
Pascoe, E and Richman, S. (2009). Perceived discrimination and health: A metaanalytic review. Psychological Bulletin, 135(4): 531-554.

Paul, K and Moser, K. (2009). Unemployment impairs mental health: Meta-analyses. Journal of Vocational Behavior, 74(3): 264-282.

Raftery, A. (1995). Bayesian model selection in social research. Sociological Methodology, 25, 111-163.

Rogers, S., Thrasher, A., Miao, Y., Boscardin, W. and Smith, A. (2015). Discrimination in healthcare settings is associated with disability in older adults: Health and retirement study, 2008-2012. Journal of General Internal Medicine, 30, 1413-20.

San Jose, J., Amado, C. (2017). On studying ageism in long-term care: A systematic review of the literature. International Psychogeriatrics, 29(3), 373-387.

Sargent-Cox, K. (2017). Ageism: we are our own worst enemy. International Psychogeriatrics, 29(1), 1-8.

Schroyen, S., Missotten, P., Jerusalem, G., Giles, C. and Adam, S. (2016). Ageism and caring attitudes among nurses in oncology. International Psychogeriatrics, 28(5), 749-757.

Temple, J. and Kelaher, M. (2018). Is disability exclusion associated with psychological distress? Australian evidence from a national cross-sectional survey. BMJ Open, 8, e020829. doi: 10.1136/bmjopen-2017-020829 
Temple, J. and Williams, R. (2018). Multiple health conditions and barriers to healthcare among older Australians: Prevalence, reasons and types of barriers. Australian Journal of Primary Health, 24(1), 82-89.

Tinney, J., Dow, B., Maude, P., Purchase, R., Whyte, C., Barrett, C. (2015). Mental health issues and discrimination among older LGBTI people. International Psychogeriatrics, 27(9), 1411-1416.

VicHealth. (2012). Disability and health inequalities in Australia - Research Summary: Addressing the social and economic determinants of mental and physical health. Melbourne: Victorian Health Promotion Foundation.

Wamala, S., Bostrom, G. and Nyqvist, K. (2007). Perceived discrimination and psychological distress in Sweden. British Journal of Psychiatry, 190, 75-76.

Werner, P and Giveon, S. (2008). Discriminatory behaviour of family physicians toward a person with Alzheimer's disease. International Psychogeriatrics, 20(4): 824-839.

World Health Organization. (2011). Global Health and Aging, NIH Publication no. 117737.

World Health Organization. (2012). Projections of mortality and causes of death: 2015 and 2030. Available at http://www.who.int/healthinfo/global burden disease/projections/en/, accessed 17/04/18.

Zou, G. (2004). A modified Poisson regression approach to prospective studies with binary data. American Journal of Epidemiology, 159(7), 702-706. 


\section{University Library}

\section{- M M N E R VA A gateway to Melbourne's research publications}

Minerva Access is the Institutional Repository of The University of Melbourne

Author/s:

Temple, JB;Kelaher, M;Williams, R

Title:

Disability discrimination and avoidance in later life: prevalence, disability differentials and association with mental health

Date:

2018-12-07

Citation:

Temple, J. B., Kelaher, M. \& Williams, R. (2018). Disability discrimination and avoidance in later life: prevalence, disability differentials and association with mental health. International Psychogeriatrics, 31 (9), pp.1319-1329. https://doi.org/10.1017/S1041610218001722.

Persistent Link:

http://hdl.handle.net/11343/253840 